\title{
Anisotropic Elastic-Viscoplastic Properties at Finite Strains of Injection-Moulded Low-Density Polyethylene
}

\author{
M. Kroon ${ }^{1}$ - E. Andreasson ${ }^{2,4}$ - E. Persson Jutemar ${ }^{2}$ • V. Petersson ${ }^{2}$. \\ L. Persson' ${ }^{2}$ M. Dorn' ${ }^{1}$ - P.A.T. Olsson ${ }^{3}$
}

Received: 7 February 2017 / Accepted: 25 July 2017 / Published online: 18 August 2017

(C) The Author(s) 2017. This article is an open access publication

\begin{abstract}
Injection-moulding is one of the most common manufacturing processes used for polymers. In many applications, the mechanical properties of the product is of great importance. Injection-moulding of thin-walled polymer products tends to leave the polymer structure in a state where the mechanical properties are anisotropic, due to alignment of polymer chains along the melt flow direction. The anisotropic elastic-viscoplastic properties of low-density polyethylene, that has undergone an injectionmoulding process, are therefore examined in the present work. Test specimens were punched out from injectionmoulded plates and tested in uniaxial tension. Three in-plane
\end{abstract}

\section{Kroon}

martin.kroon@lnu.se

E. Andreasson

Eskil.Andreasson@tetrapak.com

E. Persson Jutemar

Elin.PerssonJutemar@tetrapak.com

V. Petersson

Viktor.Petersson@tetrapak.com

L. Persson

Leo.Persson@tetrapak.com

M. Dorn

michael.dorn@1nu.se

P.A.T. Olsson

par.olsson@mah.se

1 Linnaeus University, Växjö, Sweden

2 Tetra Pak, Lund, Sweden

3 Malmö University, Malmö, Sweden

4 Blekinge Technical University, Karlskrona, Sweden material directions were investigated. Because of the small thickness of the plates, only the in-plane properties could be determined. Tensile tests with both monotonic and cyclic loading were performed, and the local strains on the surface of the test specimens were measured using image analysis. True stress vs. true strain diagrams were constructed, and the material response was evaluated using an elasticviscoplasticity law. The components of the anisotropic compliance matrix were determined together with the directionspecific plastic hardening parameters.

Keywords Polyethylene - Anisotropic - Tensile ·

Viscoplasticity · Elasticity · LDPE · Injection-moulding ·

Constitutive behaviour

\section{Introduction}

Polyethylene is a polymer of great industrial importance, and the mechanical properties of this polymer are therefore of great interest. Polyethylene normally exhibits a semicrystalline microstructure. Depending on the details of the polyethylene molecules (i.e. degree of branching, length of branches, etc), different degrees of crystallinity may be attained.

Polyethylene that solidifies in the absence of concurrent deformation of the melt forms a spherulitic microstructure, which is isotropic from a mechanical point of view [1,2]. The mechanical properties of such isotropic polyethylene have been examined in several experimental studies [e.g. 3-7].

However, there are manufacturing processes that leave the polymer in an anisotropic state. One example is injection-moulding, which is one of the most common manufacturing processes for polymer products. During the injection-moulding process, a polymer melt is forced into a 
cavity under high pressure, and the geometry of the cavity determines the shape of the final product. Often one of the dimensions of the product geometry is relatively small compared to the rest of the geometry, and this dimension may therefore be termed the thickness dimension. The mechanical properties of injection-moulded articles are markedly affected by the temperature and velocity fields that melt elements in the cavity are subjected to during the moulding cycle. Layers through the thickness with differing characteristics may therefore be identified.

During the filling stage in the injection-moulding cycle, the bulk (or core) of the polymer cools very little, but next to the mould surfaces, there will be a rapidly cooling thin skin of polymer. This skin layer is normally very thin, has virtually no preferred orientation of the polymer chains, and is normally negligible from a mechanical point of view. However, between the skin and the bulk layers, adjacent layers of polymer melt flow at different velocities and generate shear stresses, which cause the polymer chains to be aligned in the direction of polymer flow. This produces a shear layer, which is highly oriented and is said to be in a state of frozen strain [8-11]. Hence, the injection-moulded material can be expected to be anisotropic and also inhomogenous in the thickness direction.

In the present paper, we investigate the anisotropic mechanical properties of low-density polyethylene (LDPE) plates that have been created through an injection-moulding process. The material is characterized in terms of its anisotropic elastic-plastic behaviour at large strains as well as its strain-rate dependency. The paper is organized as follows: In "Continuum Mechanics Definitions", we introduce the necessary continuum mechanics entities that are needed to characterize the mechanical properties of the polymer material. In "Materials and Methods", we provide the details of the experimental procedure and the methods for evaluating the experiments. The results of the experiments are presented in "Results", and "Discussion" and "Conclusions" contain a discussion of the results and some concluding remarks.

\section{Continuum Mechanics Definitions}

\section{Kinematics}

We assume the existence of a set of orthogonal basis vectors, $\mathbf{e}_{1}, \mathbf{e}_{2}$, and $\mathbf{e}_{3}$. Let $\mathbf{X}=X_{1} \mathbf{e}_{1}+X_{2} \mathbf{e}_{2}+X_{3} \mathbf{e}_{3}$ denote the position of a point in the reference (undeformed, unloaded) configuration. Let $\mathbf{x}=x_{1} \mathbf{e}_{1}+x_{2} \mathbf{e}_{2}+x_{3} \mathbf{e}_{3}$ denote the position of the same point in the current (deformed) configuration. The displacement of the point is $\mathbf{u}=\mathbf{x}-\mathbf{X}$. In general, the current position and the displacement are functions of $\mathbf{X}$, i.e. $\mathbf{x}=\mathbf{x}(\mathbf{X})$ and $\mathbf{u}=\mathbf{u}(\mathbf{X})$.
The engineering strain tensor, $\boldsymbol{\epsilon}$, is defined by

$\boldsymbol{\epsilon}=\frac{\mathrm{d} \mathbf{u}}{\mathrm{dX}}=\frac{\mathrm{d} \mathbf{x}}{\mathrm{d} \mathbf{X}}-\mathbf{I}$,

where $\mathbf{I}$ is the identity tensor. This measure of strain, defined in equation (1), will be used in the present study. However, when we speak of the 'engineering' strain in test specimens below, we mean the local/true engineering strain in the central part of the test specimens, rather than the load-line displacement divided by total initial specimen length that is sometimes termed 'engineering strain' in the literature.

Throughout this paper, bold face symbols, such as $\boldsymbol{\epsilon}$, denote tensors. The components of such tensors are associated with an orthogonal coordinate system and are denoted by $\epsilon_{i j}$, where $i, j \in[1,2,3]$.

The principal stretches of the deformation are denoted by $\lambda_{1}, \lambda_{2}$, and $\lambda_{3}$. For an orthotropic material, where a uniaxial tensile test is performed along one of the principal directions of the material, the normal strain components may be expressed as

$\epsilon_{11}=\lambda_{1}-1, \epsilon_{22}=\lambda_{2}-1, \epsilon_{33}=\lambda_{3}-1$.

The total strain may be decomposed into elastic and plastic parts, i.e.

$\epsilon=\epsilon_{\mathrm{e}}+\epsilon_{\mathrm{p}}$.

The Jacobian of the deformation, $J=\lambda_{1} \lambda_{2} \lambda_{3}$, quantifies the volume change of the material, where $J=1$ signifies incompressibility. Plastic deformations may be assumed to be incompressible, and for cases where the plastic strains are far greater then the elastic strains, $J \approx 1$ may be assumed. For uniaxial tensile tests performed along one of the principal directions of the material, where the loading has progressed well into the plastic regime, the strain in the thickness direction, i.e. $\epsilon_{33}$, may therefore be estimated through the relation

$\epsilon_{33}=\lambda_{3}-1 \approx \frac{1}{\lambda_{1} \lambda_{2}}-1=\frac{1}{\left(1+\epsilon_{11}\right)\left(1+\epsilon_{22}\right)}-1$.

\section{Stress}

We introduce the symmetric (true) Cauchy stress tensor, $\sigma$, and the non-symmetric first Piola-Kirchhoff (or engineering) stress tensor, $\mathbf{P}$. In a uniaxial tensile test, where a force, $F$, is applied to a test specimen, the first Piola-Kirchhoff stress state is given by

$P_{11}=\frac{F}{A_{0}}$, all other $P_{i j}=0$, 
where $A_{0}$ is the initial cross-sectional area of the test specimen, and where the force is applied in the $\mathbf{e}_{1}$-direction. The true stress is given by

$\sigma_{11}=\frac{F}{A_{0} \lambda_{2} \lambda_{3}}=\frac{P_{11}}{\lambda_{2} \lambda_{3}}=\frac{P_{11} \lambda_{1}}{J}$, all other $\sigma_{i j}=0$,

where the entity $A_{0} \lambda_{2} \lambda_{3}$ is the current (true) cross-sectional area of the specimen. In the case of incompressible deformation, $J=1$ holds, implying that $\sigma_{11}=P_{11} \lambda_{1}$.

\section{Elastic Material Response}

The injection-moulding manufacturing process leaves the polyethylene material in question with three principal directions, and the material may therefore be characterized as orthotropic. The material is taken to be elastic-plastic, and both the elastic and plastic properties may be assumed to be orthotropic.

The elastic behaviour of an orthotropic material requires the determination of 9 independent elasticity constants, and the elastic behaviour can be described by the compliance matrix, according to

$$
\left[\begin{array}{c}
\epsilon_{\mathrm{e}, 11} \\
\epsilon_{\mathrm{e}, 22} \\
\epsilon_{\mathrm{e}, 33} \\
2 \epsilon_{\mathrm{e}, 12} \\
2 \epsilon_{\mathrm{e}, 13} \\
2 \epsilon_{\mathrm{e}, 23}
\end{array}\right]=\left[\begin{array}{cccccc}
\frac{1}{E_{1}} & -\frac{\nu_{21}}{E_{2}} & -\frac{\nu_{31}}{E_{3}} & 0 & 0 & 0 \\
-\frac{\nu_{12}}{E_{1}} & \frac{1}{E_{2}} & -\frac{\nu_{32}}{E_{3}} & 0 & 0 & 0 \\
-\frac{\nu_{13}}{E_{1}} & -\frac{\nu_{23}}{E_{2}} & \frac{1}{E_{3}} & 0 & 0 & 0 \\
0 & 0 & 0 & \frac{1}{G_{12}} & 0 & 0 \\
0 & 0 & 0 & 0 & \frac{1}{G_{13}} & 0 \\
0 & 0 & 0 & 0 & 0 & \frac{1}{G_{23}}
\end{array}\right]\left[\begin{array}{c}
\sigma_{11} \\
\sigma_{22} \\
\sigma_{33} \\
\sigma_{12} \\
\sigma_{13} \\
\sigma_{23}
\end{array}\right]
$$

where $E_{1}, E_{2}$, and $E_{3}$ are the Young's moduli of the three principal directions, $v_{i j}, i, j \in[1,2,3]$, are the Poisson's ratios, and $G_{12}, G_{13}, G_{23}$ are the shear moduli. Of these 12 parameters inside the $6 \times 6$ matrix, only 9 are independent, owing to the symmetry of the compliance matrix.

The three Young's moduli and the Poisson's ratios of the compliance matrix can be determined from three uniaxial tensile tests performed along the three principal directions of the material, and the shear moduli can be determined from three shear tests.

\section{Plastic Material Response}

The uniaxial plastic behaviour may be characterized by the initial yield stress, $\sigma_{\mathrm{y} 0}$, and a hardening behaviour. The hardening curve describes the evolution of the yield stress, $\sigma_{\mathrm{y}}$, as a function of $\epsilon_{\mathrm{p}}$, the plastic strain in the direction of loading during the uniaxial tensile test. A time-independent yield function, $\sigma_{\mathrm{y}}\left(\epsilon_{\mathrm{p}}\right)$, is attained for low loading rates, i.e. for $\dot{\epsilon}_{\mathrm{p}} \rightarrow 0$. In the present study, we adopt a linear hardening law, i.e.

$\sigma_{\mathrm{y}}=\sigma_{\mathrm{y} 0}\left(1+H \epsilon_{\mathrm{p}}\right)$,

where $H$ is the plastic modulus (normalized by $\sigma_{\mathrm{y} 0}$ ).

In general, the uniaxial stress, $\sigma$, required to drive plastic deformation, also depends on the deformation rate, $\dot{\epsilon}_{\mathrm{p}}$, i.e. the material is visco-plastic. In the present study, we assess the visco-plastic behaviour by use of a Johnson-Cook-like viscoplasticity law, namely

$\dot{\epsilon}_{\mathrm{p}}=\dot{\epsilon}_{0}\left(\exp \left(\frac{\sigma-\sigma_{\mathrm{y}}}{\sigma_{\mathrm{r}}}\right)-1\right)$,

which is valid for $\sigma>\sigma_{\mathrm{y}}$, where $\dot{\epsilon}_{0}$ and $\sigma_{\mathrm{r}}$ are material constants, and $\sigma$ is the current stress in the uniaxial tensile test.

For an orthotropic material, each material direction has its own initial yield stress and hardening behaviour.

\section{Materials and Methods}

\section{Material}

The tested material was a low-density polyethylene (LDPE), which came in plates that had been created through an injection-moulding process, see Fig. 1. The base material utilized was a white-pigmented LDPE with an MFI (Melt Flow Index) of approximately $20 \mathrm{~g} / 10 \mathrm{~min}\left(190{ }^{\circ} \mathrm{C}, 2.16\right.$ kg; ASTM D1238-04 and ISO 1133:1997). A standard injection-moulding machine, i.e. a horizontal Arburg 470 800-70S hydraulic injection moulding equipment, was used to manufacture the polymeric test plates, following ISO 294-5. The plastic melt, with a temperature of $240{ }^{\circ} \mathrm{C}$, was injected with a volume flow of $20 \mathrm{~cm}^{3} / \mathrm{s}$ into a tool with a temperature of $40{ }^{\circ} \mathrm{C}$. This was followed by cooling for 11 seconds. The test plates were produced by pure injection-moulding, and the flood gate ensures that the flow front evolves evenly in the test plates, see Fig. 1. The flow direction is denoted by 'MD', the direction perpendicular to the flow direction is denoted by 'CD', and the thickness direction is denoted by 'TD'. The plates had a thickness of $0.60 \mathrm{~mm}$.

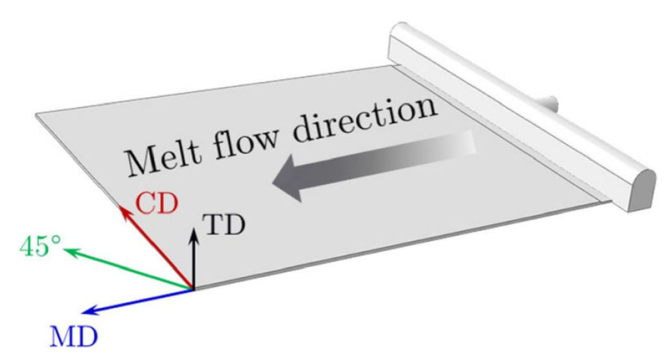

Fig. 1 Injection-moulding of test plates 

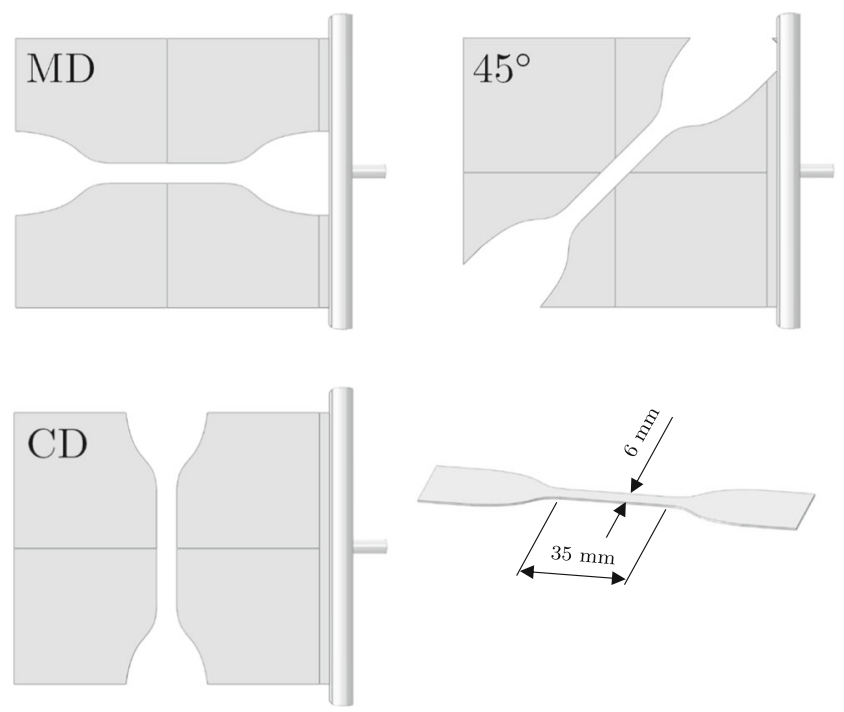

Fig. 2 Material directions of injection-moulded plates, test specimen orientations in the plates, and dimensions of the test specimens

Test specimens, suitable for tensile testing and shaped as dog bones, were punched out mechanically from the polymer plates, see Fig. 2. Three material directions were investigated, i.e. $\mathrm{MD}, \mathrm{CD}$ and an intermediate direction, $45^{\circ}$ to $\mathrm{MD}$ and $\mathrm{CD}$. The dog bone geometry utilized follows the ISO standard of the test plate. The length of the area where the local strain is measured is $35 \mathrm{~mm}$, the width of the measuring area is $6.0 \mathrm{~mm}$, and the thickness of the specimen is the same as the plate thickness, i.e. $0.60 \mathrm{~mm}$, see Fig. 2.

With regard to indices of stress and strain tensors, we will, throughout this paper, use the index convention $\mathrm{MD} \Leftrightarrow$ $\mathbf{e}_{1}, \mathrm{CD} \Leftrightarrow \mathbf{e}_{2}$, and $\mathrm{TD} \Leftrightarrow \mathbf{e}_{3}$, where $\mathbf{e}_{1}, \mathbf{e}_{2}$, and $\mathbf{e}_{3}$, constitute a set of orthogonal basis vectors.

\section{Testing Procedure}

A standard tensile testing machine was used for the tensile tests, and the testing was performed under displacement control with a prescribed grip separation velocity. All tests were performed at room temperature. The immediate output from the tensile tests was the load vs. time data. Three different loading rates (i.e. grip separation speeds) were used: $0.5 \mathrm{~mm} / \mathrm{s}, 10 \mathrm{~mm} / \mathrm{s}$, and $100 \mathrm{~mm} / \mathrm{s}$. In terms of strain rate in the central part of the test specimens, where the local strain is measured, these deformation rates correspond approximately to strain rates of $0.0125 / \mathrm{s}, 0.25 / \mathrm{s}$, and $2.5 / \mathrm{s}$, respectively. The strain rates (as well as the thickness of the plates) were chosen so that they would reflect the deformation processes that applications in the packaging industry (e.g. beverage packages) might be exposed to. For each test condition, three tests were made in order to get an impression of the statistical dispersion in the results. Most of the tests were done using a monotonically increasing displacement, but a few tests were performed with cyclic loading.

\section{Local Strain Measurement}

The tests were also video recorded using a standard video camera with a frame rate of 30 frames per second. Furthermore, the local strains on the surface of the test specimens during the tests could be established using image analysis. To facilitate this strain evaluation, lines were drawn on the specimens using a colour pen, as illustrated in Fig. 3(a).

An image analysis toolbox for Matlab was utilized. The analysis method is illustrated in Fig. 3. From the videos, snapshot images are taken with appropriate frequency, so that the straining process is properly resolved. At the highest loading rate, all 30 frames per second had to be used, in order to get a reasonable resolution of the straining process. Markers were defined in a reference image (at zero strain), see Fig. 3(a). After that, the image analysis program tracks the movement of these markers in subsequent images, which represent different degrees of specimen deformation, see Fig. 3(a).

Once the current positions of the markers have been established for a specific image, the displacement of the positions can be plotted vs. the reference positions of the
Fig. 3 Illustration of strain measurement in image analysis:

(a) Specimen with lines that can be tracked during the deformation; (b) Approximation of engineering strain using displacement of several displaced lines (a)

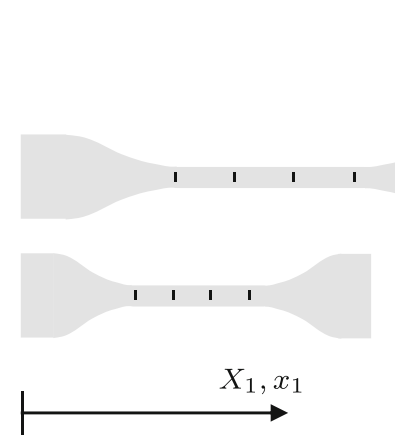

(b)

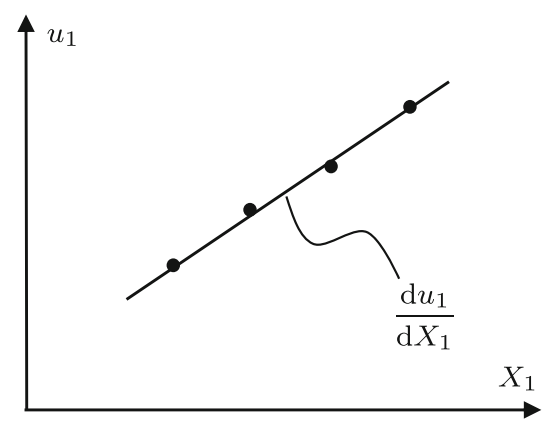


markers, see Fig. 3(b). A straight line is fitted to the marker displacements, and the slope of this fitted line provides an estimate of the engineering strain in the central part of the specimen, in this case $\epsilon_{11}=\mathrm{d} u_{1} / \mathrm{d} X_{1}$.

The markers in Fig. 3(a) illustrate the measurement of strain in the loading direction, but markers were used in the same way for measuring the strain in the direction perpendicular to the loading direction. Hence, both the strain in the loading direction and that in the direction perpendicular to loading could be measured.

\section{Evaluation of Elastic-plastic Behaviour}

In the present study, we only measured the in-plane elastic properties of the injection-moulded LDPE plates. Hence, only $E_{1}, E_{2}, v_{12}, v_{21}$, and $G_{12}$ were determined. The first four of these constants were determined from the uniaxial tensile tests in $\mathrm{MD}$ and $\mathrm{CD}$. However, $G_{12}$ was not determined from a shear test but from the tensile tests performed in the $45^{\circ}$ direction. The shear modulus $G_{12}$ may be expressed as

$G_{12}=\frac{1}{\frac{4}{E_{45}}-\frac{1}{E_{1}}-\frac{1}{E_{2}}+\frac{\nu_{21}}{E_{2}}+\frac{\nu_{12}}{E_{1}}}$,

where $E_{45}$ is the Young's modulus for the $45^{\circ}$ direction.

It should be emphasized, that in the derivations above, we have assumed that deformations are confined to the elastic regime and that strains and any possible rotations of the material therefore remain small.

Just as for the elastic properties, the plastic properties of the three in-plane directions were determined in the experiments. Hence, the initial yield stress and plastic modulus for these three directions are denoted by $\sigma_{\mathrm{y} 0,1}, H_{1}, \sigma_{\mathrm{y} 0,2}$, $\mathrm{H}_{2}, \sigma_{\mathrm{y} 0,45}$, and $\mathrm{H}_{45}$ for the $\mathrm{MD}, \mathrm{CD}$ and $45^{\circ}$ directions, respectively. The associated plastic strains and current yield stresses are denoted by $\epsilon_{\mathrm{p}, 11}, \sigma_{\mathrm{y}, 1}, \epsilon_{\mathrm{p}, 22}, \sigma_{\mathrm{y}, 2}, \epsilon_{\mathrm{p}, 45}$, and $\sigma_{\mathrm{y}, 45}$, respectively.
During a tensile test in MD, for instance, the total strain rate, $\dot{\epsilon}_{11}$, may then be expressed as

$$
\begin{aligned}
\dot{\epsilon}_{11}= & \dot{\epsilon}_{\mathrm{e}, 11}+\dot{\epsilon}_{\mathrm{p}, 11}=\frac{\dot{\sigma}_{11}}{E_{1}}+\dot{\epsilon}_{\mathrm{p}, 11}, \\
\dot{\epsilon}_{\mathrm{p}, 11}= & \dot{\epsilon}_{0}\left(\exp \left(\frac{\sigma_{11}-\sigma_{\mathrm{y}, 1}\left(\epsilon_{\mathrm{p}, 11}\right)}{\sigma_{\mathrm{r}}}\right)-1\right) \\
& \cdot \Theta\left(\sigma_{11}-\sigma_{\mathrm{y}, 1}\left(\epsilon_{\mathrm{p}, 11}\right)\right) \\
\epsilon_{\mathrm{p}, 11}= & \int_{t} \dot{\epsilon}_{\mathrm{p}, 11} \mathrm{~d} t
\end{aligned}
$$

where $\Theta(\bullet)$ is the Heaviside step function, which takes on the value 1 for $(\bullet)>0$ and zero otherwise.

In the experiments, a constant deformation rate is applied, which translates to a strain rate in the central section of the specimen that is essentially constant. Hence, $\dot{\epsilon}_{11}$ may be taken to be constant during the test. Equations (11)-(13) constitute a set of differential equations that can be solved numerically for $\sigma_{11}(t)$ and $\epsilon_{\mathrm{p}, 11}(t)$. The solution also enables the prediction of the elastic-viscoplastic response, i.e. $\sigma_{11}$ vs. $\epsilon_{11}$, of the material, which may then be compared with the experimental response. Corresponding equations may be formulated and solved for the $\mathrm{CD}$ and $45^{\circ}$ directions.

The stress-strain response predicted by equations (11) (13) is compared to the stress-strain response from the experiments, and in this way, the elastic and plastic properties for the three in-plane material directions are assessed. The estimation of Young's modulus, Poisson's ratio, and plastic properties from the experiments is illustrated in Fig. 4. The estimation of $E_{1}, \sigma_{\mathrm{y} 0,1}$, and $H_{1}$ is provided as an example in Fig. 4(a). In a similar way, estimates of the Young's moduli and plastic properties of the CD and $45^{\circ}$ directions are attained.

The estimation of Poisson's ratio is illustrated in Fig. 4(b). The evolution of $\epsilon_{11}$ and $\epsilon_{22}$ with time is shown. By use of strain data $\epsilon_{11} \in[0,0.04]$ (and the corresponding data points for $\epsilon_{22}$ ), straight lines are fitted, as shown in Fig. 4(b). The slopes of these lines are denoted by $k_{1}$
Fig. 4 Estimation of elastic and plastic properties from tensile tests: (a) Estimation of Young's modulus, initial yield stress, and hardening through comparison of experimental response (solid line) and model calibration (dashed line); (b) Estimation of Poisson's ratio through fitting of two straight lines to the two strain paths (a)
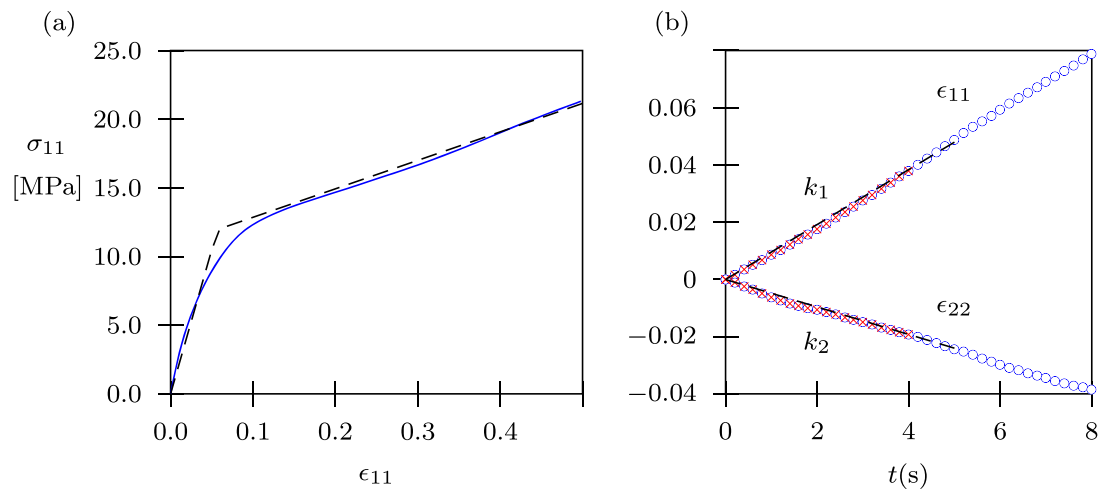
Fig. 5 Example of output from experiment in MD: (a) Force vs. time data; (b) $\epsilon_{11}$ (blue), $\epsilon_{22}$ (red), and $\epsilon_{33}$ (dashed green) vs. time data

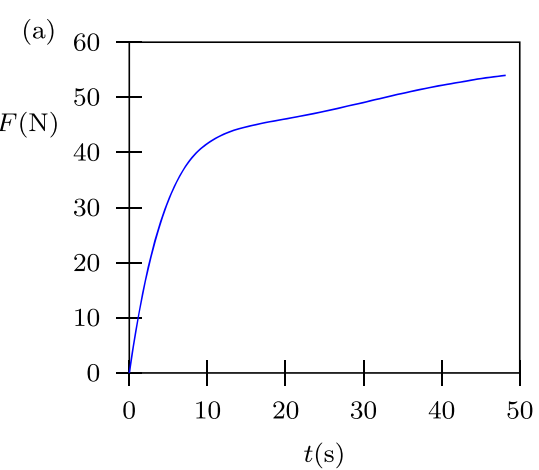

and $k_{2}$, and the value of Poisson's ratio is then estimated as

$v_{12} \approx-\frac{k_{2}}{k_{1}}$.

In a similar way, estimates of $v_{21}$ and $v_{45}$ (the Poisson's ratio associated with stretching in the $45^{\circ}$ direction) are attained.

\section{Results}

\section{Force and Strain Data from Experiments}

Figure 5 shows the output from a test for MD at a deformation rate of $0.5 \mathrm{~mm} / \mathrm{s}$. The force data was filtered from noise.

The force data in Fig. 5(a) shows a monotonically increasing force. This was only seen for MD. The force data for the $\mathrm{CD}$ and $45^{\circ}$ directions showed a decreasing force once the onset of plastic deformation had occurred. The true stress vs. strain relation, however, always showed a monotonically increasing stress, i.e. no true material softening was observed.

In Fig. 5(b) the associated strain data is shown. The strain in the loading direction, $\epsilon_{11}$, shows an almost linear increase with time. The strain in $\mathrm{CD}, \epsilon_{22}$, also varies essentially linearly with time, but with a negative slope. The ratio $\epsilon_{22} / \epsilon_{11}$ allows for a determination of the Poisson's ratio $v_{12}$.

In Fig. 5(b), the estimation of $\epsilon_{33}$, according to equation (4), is also included. In this case, the curve for $\epsilon_{33}$ virtually overlaps the curve for $\epsilon_{22}$. This means that the plastic strains in $\mathrm{CD}$ and TD, that are caused by the plastic stretching in $\mathrm{MD}$, are distributed equally to $\mathrm{CD}$ and TD.

\section{Strain Response During Monotonic Loading}

In Figs. 6, 7, and 8, the outcome from the strain measurements is shown for the three different loading rates, respectively.

Starting with the slowest loading rate in Fig. 6, we note that there is some discrepancy between the strain histories of the different directions. The total elongation of the test specimens is distributed between the thin area of local strain measurement and the wider parts where the specimen is clamped. This distribution appears to differ between the different directions, which causes the observed discrepancies in strain history. The Poisson's ratios, $v_{12}$ and $v_{21}$, were calculated for each test using equation (14).

The $45^{\circ}$ direction was the direction that exhibited the most ductility in the sense that the highest fracture/localization strains were observed for this direction.
Fig. 6 Strain vs. time data from experiments for $\dot{\epsilon}=0.0125 / \mathrm{s}$. Solid lines: strain in loading direction; dashed and dotted lines: strain in the transverse and thickness directions, respectively

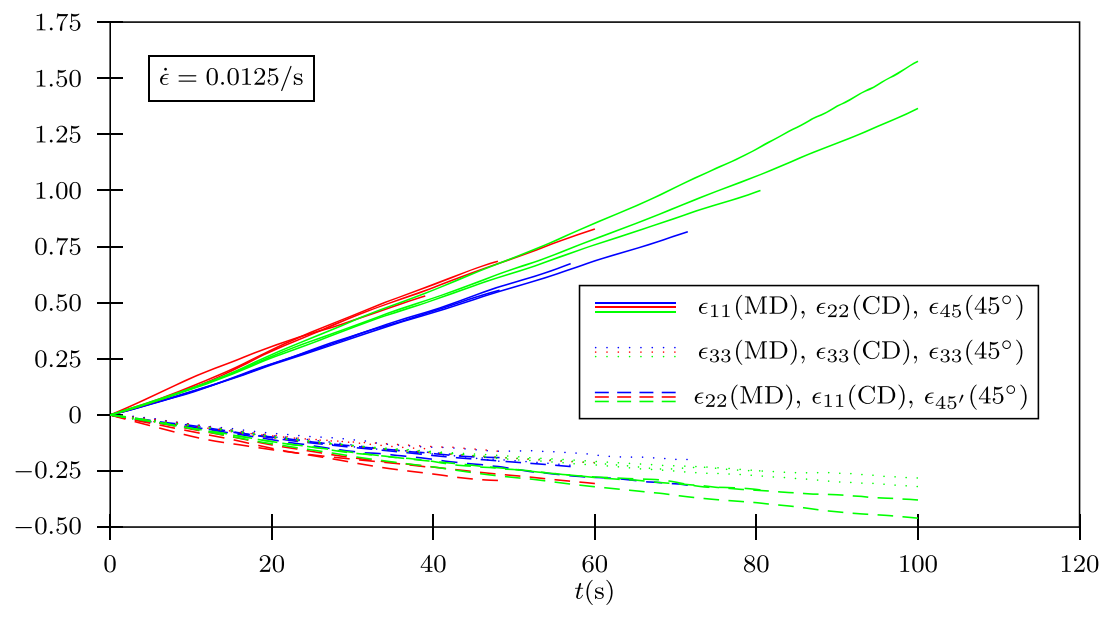


Fig. 7 Strain vs. time data from experiments for $\dot{\epsilon}=0.25 / \mathrm{s}$. Solid lines: strain in loading direction; dashed and dotted lines: strain in the transverse and thickness directions, respectively

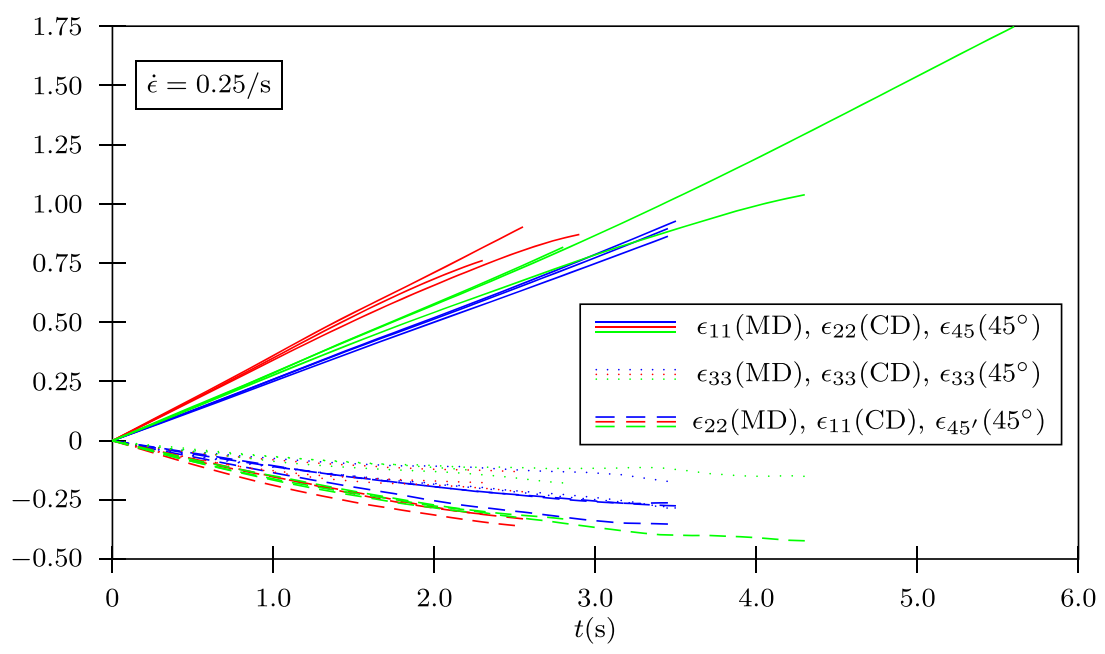

This must be attributed to the microstructure of the injection-moulded material. The in-plane strain perpendicular to the $45^{\circ}$ direction is denoted by $\epsilon_{45^{\prime}}$.

The central part of the test specimens (where the strain measuring takes place) for the $45^{\circ}$ did not undergo a simple stretching during the tests. The material is anisotropic, and when a tensile test is performed in a direction that does not coincide with one of the principal directions of the material, the material will undergo a deformation consisting of both stretching and shearing. However, the shearing was not measured during the present tests.

In Figs. 7 and 8, the outcomes from the tests at the medium and highest loading rates are displayed. Again, there is a systematic discrepancy in the local true strain history between the different directions. The tendency is again that for the $\mathrm{CD}$ direction, more of the total strain is distributed to the thin area of measurement compared to the other directions. It may also be noted, that the difference between the strains in the perpendicular directions is more pronounced compared to what was observed at the slowest loading rate.

\section{Stress-strain Response During Monotonic Loading}

Based on the force vs. time and strain vs. time data, the stress-strain response could be established. Since we were not able to measure the strain in TD, we assume incompressibility for all deformations, i.e. also for the elastic deformations. This assumption is necessary to enable the computation of the true stress in the test specimens.

Figure 9 shows the stress-strain results for monotonic loading in MD. The individual coloured lines indicate individual tests. The initial elastic response is similar for the three loading rates, even though a slight non-linearity in the initial response can be identified for the lower loading rates. The initial elastic response is taken to be rate-independent, and Young's modulus is therefore estimated as the average of all the initial responses from the tests.
Fig. 8 Strain vs. time data from experiments for $\dot{\epsilon}=2.5 / \mathrm{s}$. Solid lines: strain in loading direction; dashed and dotted lines: strain in the transverse and thickness directions, respectively

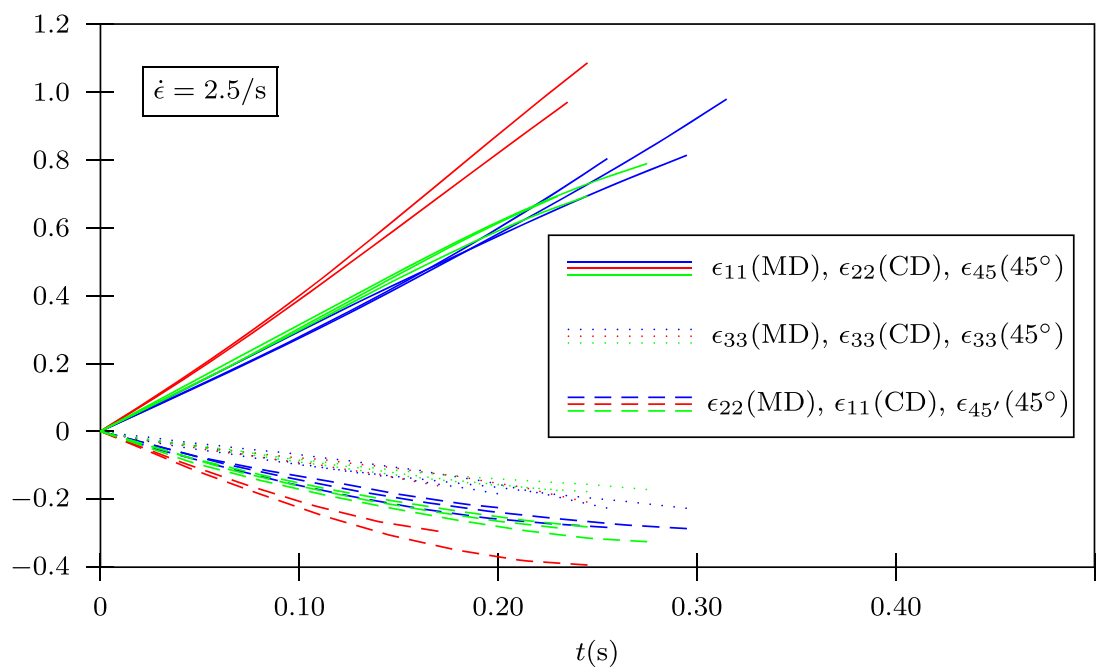




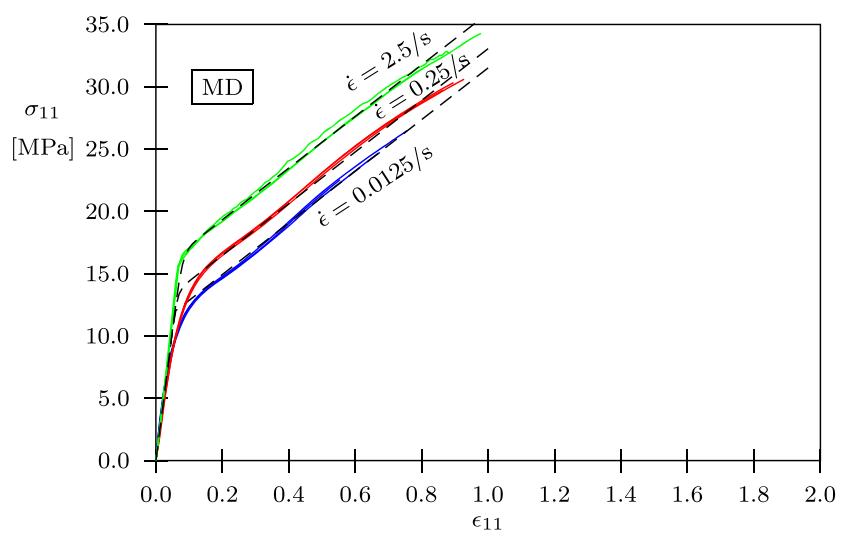

Fig. 9 Stress strain data from experiments for MD (blue: $\dot{\epsilon}=$ $0.0125 / \mathrm{s}$, red: $\dot{\epsilon}=0.25 / \mathrm{s}$, and green $: \dot{\epsilon}=2.5 / \mathrm{s}$ ) together with model prediction (dashed black line)

The plastic hardening is remarkably linear, and the curves for the three loading rates are virtually parallel. The stress required to drive plastic deformation increases with loading rate, which is clear evidence of the presence of strainrate effects in the material. The proposed hardening and visco-plasticity laws are able to represent the experimental outcome very well.

Figure 10 shows the outcome for CD. In this direction, there is a pronounced initial non-linearity, at least for the lowest loading rate. Just as in the case for MD, Young's modulus is taken to be the average for the three loading rates. In addition, the initial yielding behaviour in $\mathrm{CD}$ differs from the yielding behaviour of MD. In MD, the hardening is fairly constant after the onset of plastic yielding, whereas in $\mathrm{CD}$, the hardening decreases slightly after onset of plastic yielding, and then after that, the hardening increases again and becomes fairly constant. A consequence of this peculiarity is that the model predictions for CD are relatively

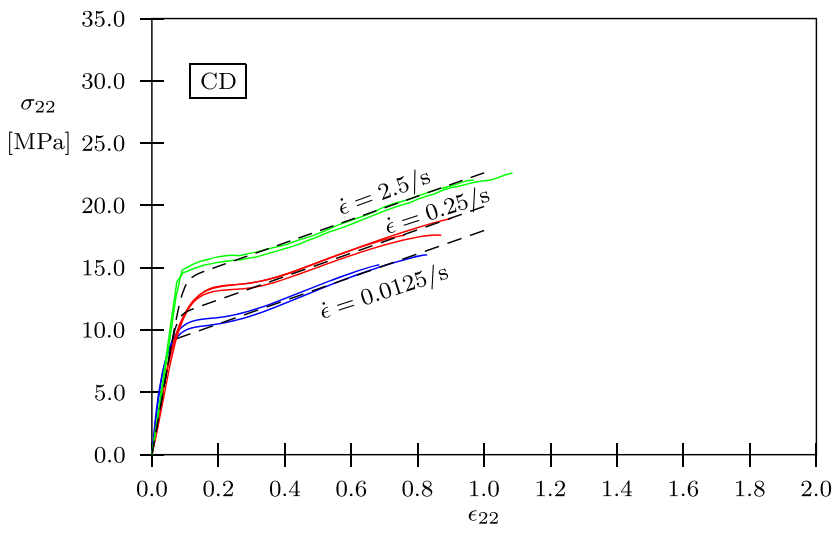

Fig. 10 Stress strain data from experiments for $\mathrm{CD}$ (blue: $\dot{\epsilon}=$ $0.0125 / \mathrm{s}$, red: $\dot{\epsilon}=0.25 / \mathrm{s}$, and green: $\dot{\epsilon}=2.5 / \mathrm{s}$ ) together with model prediction (dashed black line) inaccurate in the regime where plastic deformation is initiated, but the prediction becomes better once the constant hardening regime is entered.

Figure 11 shows the results for the $45^{\circ}$ direction. The $45^{\circ}$ direction was the direction that showed the most ductility, and the material could be strained up to almost $200 \%$ before failure occurred. The plastic hardening behaviour is fairly linear up to about $100 \%$ of straining. After that, the hardening decreases slightly. As a result, the model predictions of the viscoplastic response become increasingly inaccurate at the highest strains.

In Table 1, direction-specific material constants characterizing the elastic and plastic behaviour of the material are tabulated. Hence, the Young's modulus, the initial yield stress, the plastic modulus, and the visco-plasticity parameters are listed for the three in-plane directions that were tested.

The elastic properties can be given either in terms of stiffness or compliance. In our case, it is convenient to characterize the initial response of the material in terms of material constants associated with the compliance matrix in equation (7). The compliance matrix is a $6 \times 6$ matrix, whose components we denote by $S_{i j}$. Hence, we have $S_{12}=$ $-v_{12} / E_{1}=S_{21}=-v_{21} / E_{2}$, see equation (7). Thus, we provide estimates of the stiffness values $E_{1}, E_{2}$, and $G_{12}$ together with the component $S_{12}\left(=S_{21}\right)$ from the compliance matrix. Based on these estimates, the values of $v_{12}$ and $v_{21}$ may be calculated if needed.

The elastic constants were estimated as follows. The Young's modulus $E_{1}$ was fitted to the initial elastic slope of the test data for MD, as illustrated in Fig. 4. The same procedure was used to establish $E_{2}$ and $E_{45}$ from the tests in the $\mathrm{CD}$ and $45^{\circ}$ directions, respectively. For each test in $\mathrm{MD}$ and $\mathrm{CD}$, the values of $v_{12}$ and $\nu_{21}$ could also be extracted. The associated values $S_{12}=-v_{12} / E_{1}$ and $S_{21}=-v_{21} / E_{2}$ could then be computed, since $E_{1}$ and $E_{2}$ had already been

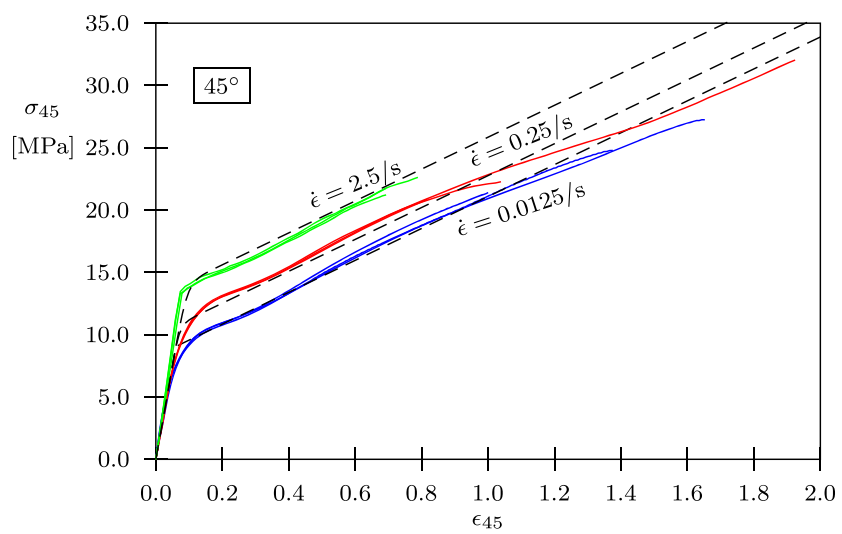

Fig. 11 Stress strain data from experiments for $45^{\circ}$ direction (blue: $\dot{\epsilon}=0.0125 / \mathrm{s}$, red $: \dot{\epsilon}=0.25 / \mathrm{s}$, and green: $\dot{\epsilon}=2.5 / \mathrm{s}$ ) together with model prediction (dashed black line) 
Table 1 Direction-specific material constants from uniaxial tensile tests

\begin{tabular}{llllll}
\hline Direction & $E$ & $\sigma_{\mathrm{y} 0}$ & $H$ & $\dot{\epsilon}_{0}$ & $\sigma_{\mathrm{r}}$ \\
& {$[\mathrm{MPa}]$} & {$[1 / \mathrm{s}]$} & 0.1 & 1.6 \\
\hline $\mathrm{MD}$ & 210 & 12 & 23 & 0.05 & 1.35 \\
$\mathrm{CD}$ & 150 & 9 & 10 & 0.1 & 1.7 \\
$45^{\circ}$ & 150 & 9 & 14 & & \\
\hline
\end{tabular}

determined. Hence, each test in MD and CD produced one estimate of $S_{12}$ and $S_{21}$, respectively. The final estimate of $S_{12}$ was then taken to be the average of all values of $-v_{12} / E_{1}$ and $-v_{21} / E_{2}$ measured from the tests. Based on the estimates of $E_{1}, E_{2}, E_{45}$, and $S_{12}$, the value of $G_{12}$ was then computed using equation (10). The elastic constants thus attained are listed in Table 2.

It should be noted, that the dispersion in results for $S_{12}$ and $S_{21}$ was relatively high with a standard deviation of about 0.68 . The estimation of $S_{12}$ should therefore be regarded as highly approximate. The reason for this uncertainty is most likely that the distance used for measuring the transverse contraction (i.e. the width of the test specimens) was relatively small.

\section{Failure Strains and Failure Modes}

As can be observed from Figs. 6-11, there seems to be a systematic variation in failure strain. For MD, the failure strain increases with loading rate from about 0.6 (on the average) at the lowest loading rate up to 0.9 (on the average) at the highest rate. For $\mathrm{CD}$, the failure strain also increases with loading rate from about 0.75 to 1.0 . The $45^{\circ}$ direction is by far the most ductile direction. Interestingly, for this direction, the failure strain shows no consistent pattern. That is, the highest loading rate is associated with the lowest failure strain (about 0.7), whereas the lower loading rates are associated with the highest failure strains, ranging from 1.0 up to 1.9 .

It should be noted, that the end of the strain history in the tests was not always caused by final failure, but in a few tests, local neck formation in the measuring area prohibited further measurements of the local strain history.

In Fig. 12, some typical fracture surfaces are shown. As can be seen from both Fig. 12(a) and (b), failure in MD and CD occurred more or less perpendicularly to the loading direction and at a well defined failure site. On the other hand, failure in the $45^{\circ}$ direction occurred in a zone that was smeared out and approximately oriented $45^{\circ}$ to the loading direction. These observations were made at all loading rates.

\section{Stress-strain Response During Cyclic Loading}

In addition to the tensile tests with monotonic loading, we also did two tests with cyclic loading, one for MD and one for $\mathrm{CD}$. The purpose of these cyclic tests was primarily to get at least a qualitative impression of the material performance during unloading. The outcome of these tests is shown in Figs. 13 and 14.

In Fig. 13, the response for MD is shown. Figure 13(a) shows an overview of the stress-strain response for the cyclic load case, and Fig. 13(b) shows a close-up of the unloading process. As can be seen in Fig. 13(b), three unloadings are made at the beginning of the plastic regime. From the hysteresis of the unloading/reloading curves, it is obvious that the material response contains some additional viscosity that is not incorporated in the elastic-viscoplastic model that was used to assess the material behaviour. It is also worth noting, that the general hardening behaviour of the curve with unloading/reloading essentially follows the curves for monotonic loading, although the unloading/reloading process seems to slightly weaken the material response. On the other hand, this discrepancy could also be due to statistical variations in the material response.

In Fig. 14(a) and (b), the result of unloading/reloading for CD is shown, where Fig. 14(b) contains a close-up of the cyclic loading process. Again it is evident that the general hardening behaviour of the curve with unloading essentially follows the same behaviour as the curves with monotonic loading.

\section{Discussion}

In the present experimental study, we have examined the inplane, anisotropic, elastic-viscoplastic properties of LDPE
Table 2 Estimated in-plane elastic properties

\begin{tabular}{llll}
\hline$E_{1}$ & $E_{2}$ & $S_{12}$ & $G_{12}$ \\
{$[\mathrm{MPa}]$} & {$[\mathrm{MPa}]$} & {$[1 / \mathrm{GPa}]$} & {$[\mathrm{MPa}]$} \\
\hline 210 & 150 & -3.0 & 46 \\
\hline
\end{tabular}


Fig. 12 Examples of fracture surfaces for the three test directions; (a) MD, (b) CD, (c) $45^{\circ}$ (a)

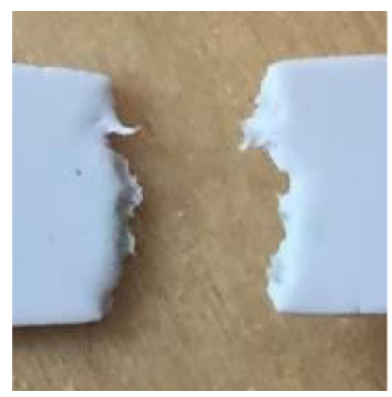

(b)

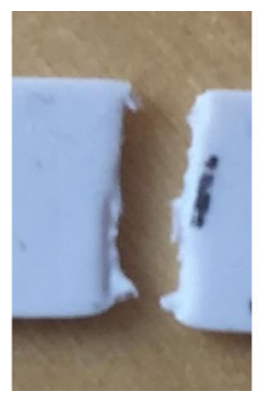

(c)

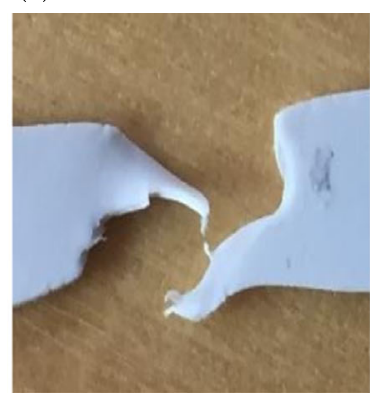

that has undergone an injection-moulding process. Test specimens were punched out from plates, and because of the small thickness of the plates, only the in-plane properties could be determined.

$\mathrm{MD}$ and $\mathrm{CD}$ are the principal material directions within the plane of the plates investigated. The stress-strain response of the present polymer differs from what is commonly observed for ductile, low molecular weight materials. The transition from initial elastic to subsequent plastic deformation was smooth, and a specific yield strain was difficult to identify. The initial response was nonlinear, especially at the lower loading rates. The fact that the initial response differed between different loading rates is also an indication that there are additional viscous/viscoelastic effects that are not properly accounted for by the elastic-viscoplastic model adopted for the assessment. Furthermore, the non-linear initial response is most likely associated with the deformation of the amorphous sections in combination with rotation of the crystalline segments of the semi-crystalline microstructure of the LDPE material.

The plastic hardening behaviour, on the other hand, was remarkably linear in the stress vs. strain diagrams. Hence, the viscoplastic behaviour could be well described by a linear hardening law for the inviscid plastic response in combination with a Johnson-Cook-like viscoplasticity law. The plastic behaviour of this type of semi-crystalline material is primarily related to the deformation of the crystalline segments of the microstructure. Moderate plastic deformations are associated with dislocation motion within the crystallites, and further plastic deformation causes disruption of the crystalline segments [12-17].

The material response was assessed by the use of an elastic-viscoplastic model. Overall, this model was able to reproduce the material response well, in particular when the material was exposed to monotonic loading. It was evident, though, that there are additional features of the material response that are not fully represented by such a model. This was especially clear from the cyclic loading tests, where it was evident that there were additional viscous/viscoelastic aspects of the material response that are not accurately captured by elastic-viscoplasticity alone.

It is evident from the stress-strain diagrams, that the material is strongly anisotropic. This pertains both to the elastic and plastic behaviour. The Young's modulus and initial yield stress are about $25 \%$ higher for MD compared with $\mathrm{CD}$, and the hardening is also clearly higher for MD. Furthermore, it is clear that the material exhibits a strong strain rate-dependence. When comparing the driving stress at the lowest and highest deformation rates, the difference was about $5 \mathrm{MPa}$ for all directions, which amounts to a substantial fraction of the applied stress.
Fig. 13 Stress-strain data for cyclic test (red line) in MD $(\dot{\epsilon}=0.0125 / \mathrm{s})$. The monotonic response (blue lines) is included as a reference. (a) The whole load history; (b) Close-up of unloading/reloading regime
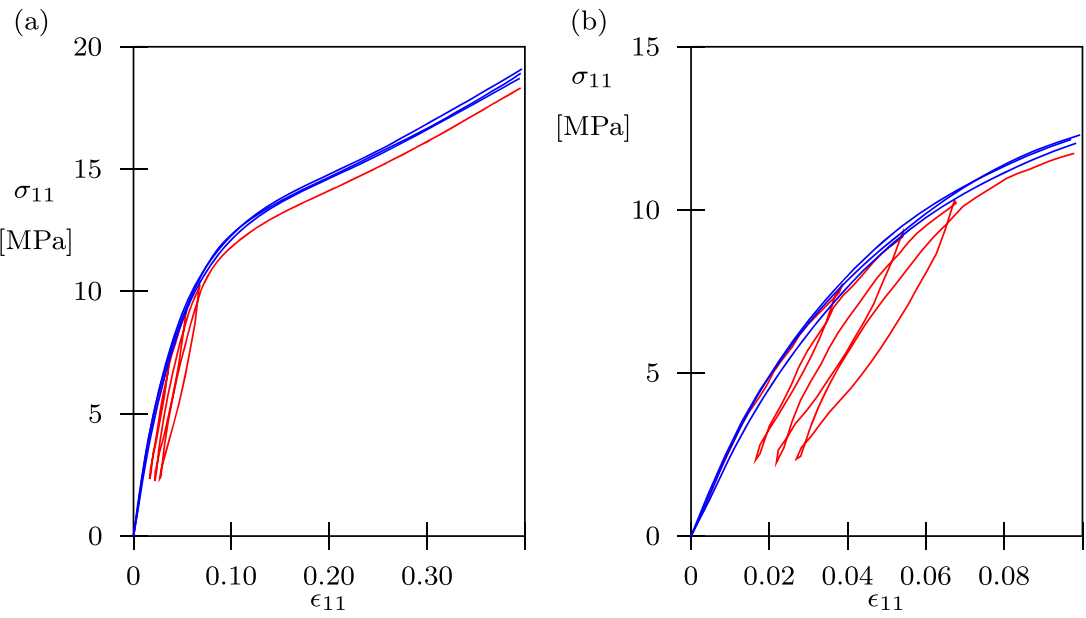
Fig. 14 Stress-strain data for cyclic test (red line) in $\mathrm{CD}$ $(\dot{\epsilon}=0.0125 / \mathrm{s})$. The monotonic response (blue lines) is included as a reference. (a) The whole load history; (b) Close-up of unloading/reloading regime

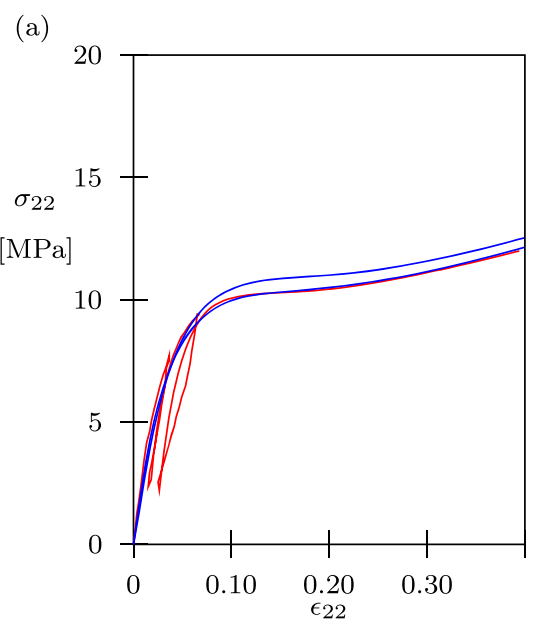

In this study, we consider the homogenized (through the thickness) behaviour of the LDPE plates. One motivation for this is that these types of thin plates are often modelled as shells rather than 3D structures. However, from a material mechanics point of view it is still of interest to characterize the mechanical properties of the different layers of these injection-moulded plates. Hence, further studies are needed, in which the microstructural features of the different layers of the plates are more accurately connected to the macroscopic material behaviour.

At present, we lack detailed knowledge of the microstructure of the injection-moulded material at hand. All three directions tested exhibited a significant amount of ductility. However, it was evident that the $45^{\circ}$ direction by far was the most ductile direction. Hence, it seems that the crystalline structure in the material is arranged in such a way that the deformation mechanisms favour plastic deformation along the $45^{\circ}$ direction.

It should also be noted, that when tests were made for the $45^{\circ}$ direction, the deformation of the thin section of the test specimens did not undergo a simple stretching, as was the case when testing was done in MD and CD. Instead, the material underwent a combined stretching and shearing deformation. This is an outcome that is to be expected when an anisotropic material is stretched in a direction that does not coincide with one of the principal material directions. The strain entity $\epsilon_{45}$ should therefore, strictly speaking, not be regarded as the normal strain in the $45^{\circ}$ direction (except for at small strains) but rather as an equivalent strain quantifying the combined stretching and shearing experienced by the material.

It is of interest to study failure strains as a function of material direction and loading rate. It was noted in the tests, that the statistical dispersion in the failure strains (or in a few cases neck formation strains) is large. Final failure is most likely governed by inhomogeneities and irregularities in the material, and it would require a much larger number of tests if the failure strains were to be determined with statistical significance.

\section{Conclusions}

The anisotropic elastic-plastic properties of injectionmoulded LDPE has been investigated. Tensile tests with both monotonic and cyclic loading were performed, and the local strains on the surface of the test specimens were measured using image analysis. True stress vs. true strain diagrams were constructed, and the material response was evaluated using an elastic-viscoplasticity law. The components of the anisotropic compliance matrix were determined together with the direction-specific plastic hardening parameters.

Acknowledgements The authors acknowledge the support from The Knowledge foundation in Sweden, grant no. 20150165.

Open Access This article is distributed under the terms of the Creative Commons Attribution 4.0 International License (http:// creativecommons.org/licenses/by/4.0/), which permits unrestricted use, distribution, and reproduction in any medium, provided you give appropriate credit to the original author(s) and the source, provide a link to the Creative Commons license, and indicate if changes were made.

\section{References}

1. Katti SS, Schultz JM (1982) The microstructure of injectionmolded semicrystalline polymers: a review. Polym Eng Sci 22:1001-1017

2. Regrain C, Laiarinandrasana L, Toillon S, Sai K (2009) Multimechanism models for semi-crystalline polymer: constitutive relations and finite element implementation. Int J Plast 25:1253-1279

3. O'Connor DG, Findley WN (1962) Influence of normal stress on creep in tension and compression of polyethylene and rigid polyvinyl chloride copolymer. SPE Trans 273-284 
4. Spitzig WA, Richmond O (1979) Effect of hydrostatic pressure on the deformation behaviour of polyethylene and polycarbonate in tension and in compression. Polym Eng Sci 19:1129-1139

5. Pawlak A, Galeski A (2005) Plastic deformation of crystalline polymers: the role of cavitation and crystal plasticity. Macromolecules 38:9688-9697

6. Drozdov AD, Christiansen JC (2008) Thermo-viscoelastic and viscoplastic behaviour of high-density polyethylene. Int J Solids Struct 45:4274-4288

7. Clausen AH, Polanco-Loria M, Berstad T, Hopperstad OS (2011) A constitutive model for thermoplastics with some applications. In: 8th European LS-DYNA users conference. Strasbourg

8. Bakerdjian Z, Kamal MR (1977) Distribution of some physical properties in injection-molded thermoplastic parts. Polym Eng Sci 17:96-100

9. Fujiyama M, Awaya H, Kimura S (1977) Mechanical anisotropy in injection-molded polypropylene. J Appl Polym Sci 21:3291-3309

10. Moy FH, Kamal MR (1980) Crystalline and amorphous orientations in injection molded polyethylene. Polym Eng Sci 20:957964
11. Tan V, Kamal MR (1978) Morphological zones and orientation in injection-molded polyethylene. J Appl Polym Sci 22:23412355

12. Addiego F, Dahoun A, G'Sell C, Hiver JM (2006) Characterization of volume strain at large deformation under uniaxial tension in high-density polyethylene. Polymer 47:4387-4399

13. Argon AS, Galeski A, Kazmierczak T (2005) Rate mechanisms of plasticity in semi-crystalline polyethylene. Polymer 46:11,79811,805

14. Bartczak Z, Argon ZS, Cohen RE (1994) Texture evolution in large strain simple shear deformation of high density polyethylene. Polymer 35:3427-3441

15. Galeski A, Bartczak Z, Argon AS, Cohen RE (1992) Morphological alterations during texture-producing plastic plane strain compression of high-density polyethylene. Macromolecules 25:5707-5718

16. Li DS, Garmestani H, Alamo RG, Kalidindi SR (2003) The role of crystallinity in the crystallographic texture evolution of polyethylenes during tensile deformation. Polymer 44:5355-5367

17. Lin L, Argon AS (1994) Review: structure and plastic deformation of polyethylene. J Mat Sci 29:294-323 